\title{
Study on Data Fusion Algorithms of Landsat7 ETM+ PAN and Multi-spectral Imagery
}

\author{
Zhihui Wang, Ximin Cui, Debao Yuan, Guo Wang, Kai Kang \\ College of Geoscience and Surveying Engineering, CUMT, Beijing, China, 100083
}

\begin{abstract}
Fusion of images with different spatial resolution can improve visualization of the images involved. This study tries to show that the fusion of the images from the same sensor system can improve quality of the original images. Four image fusion algorithms were used in the study of data fusion of Landsat 7 ETM+ imagery, taking southeastern part of Beijing City as the case study, they are the Smoothing Filter-Based Intensity Modulation (SFIM), High-Pass Filter (HPF) Transform, Brovey Transform, Multiplication (MLT) Transform. The effectiveness of the four fusion algorithms has been evaluated based on mean, deviation, information entropy, average gradient and correlation. The study reveals that the SFIM transform is the best method in retaining spectral information of original image, which does not cause spectral distortion and it has highest spatial frequency information. Therefore, fused images from the same sensor system can be used for improving visual interpretation and data quality.
\end{abstract}

Index Terms: Data fusion algorithm; PAN and multi-spectral; algorithm evaluation; ETM+

(C) 2012 Published by MECS Publisher. Selection and/or peer review under responsibility of the International Conference on E-Business System and Education Technology

\section{Introduction}

As the rapid development of information technology, sensor technology and wide application of earth observation from different satellite sensors-visible, near infrared, shortwave infrared, thermal infrared, microwave, the number of remote sensing images acquired form same region is increasing and very huge. Therefore, how to effectively utilize huge amounts of data from different sensors, different time and different resolution remote sensing image has become a popular research topic of image processing. In order to avoid parameters differences existing in different sensors and different time data and registration errors in the process of integration, Some scholars have studied panchromatic and multi-spectral image fusion from multi-resolution sensor, and made some promising results, but efforts about this research should be further strengthened.

Landsat7 was launched by the National Aeronautics and Space Bureau and carried the theme enhanced imaging sensor (ETM+), it maintained the multi-spectral characteristics of Landsat5 TM and spatial resolution

* Corresponding author.

E-mail address: Wzh8588@yahoo.com.cn 
of thermal infrared band increased from $120 \mathrm{~m}$ to $60 \mathrm{~m}$, and also added a $15 \mathrm{~m}$ panchromatic band. In view of the advantages of Landsat7 ETM+, it has become the one of most commonly used remote sensing data source, and widely used in agriculture, forest and grassland resources survey, land use and mapping, geology, hydrology, marine resources survey and environmental monitoring. Research has shown that if panchromatic and multi-spectral data can be effectively used, the accuracy of image interpretation, automatic classification and thematic mapping will be significantly improved. In this paper, 4 different fusion algorithms were applied in the fusion of panchromatic and multispectral data from landsat7 ETM+, and were analyzed and compared from quantitative aspect for taking full advantage of the landsat7 ETM+ and laying the foundation for further mining potential of landsat7 ETM+

\section{Basic principles and algorithms}

Multi-source remote sensing image fusion can generally be divided into pixel level, feature level and decision level. In this paper, spatial filtering transform(SFIM, HPF) and the algebraic transform (Brovey, MLT) as the main images fusion algorithm.

\subsection{SFIM Transform}

Smoothing Filter-based Intensity Modulation (Liu, 2000) is the transformation of brightness based on smoothing algorithm, the fusion algorithm using the following equation:

$B_{S F I M_{i}}=\sum_{i} \sum_{k} \frac{B_{\text {low }_{j k}} \times B_{\text {high }_{j k}}}{B_{\text {mean }_{j k}}}, \quad i=1, \cdots, 6$

and $B_{S F I M}$ is a fusion image created by the algorithm, $i$ is band number, $j$ and $k$ is row and column of images respectively; $B_{\text {low }}$ is low-resolution images, that is six multi-spectral bands with $30 \mathrm{~m}$ from $\mathrm{ETM}+; B_{h i g h}$ is high resolution image, that is panchromatic band with $15 \mathrm{~m} ; B_{\text {mean }}$ is simulated low-resolution images obtained by low-pass filter on the Pan band. In this study, a $5 \times 5$ filter window was used to generate $B_{\text {mean }}$ images.

PAN band filtered by low-pass filter ( $B_{\text {mean }}$ ) removed high frequency information of the band, but retained its low frequency spectral information. The ratio of $B_{\text {mean }}$ and PAN band with high resolution can offset the low-frequency spectral image and topographic contrast information, and only retain the structure and texture information of high resolution image, and then integrated high frequency information into the low resolution image. Therefore, SFIM algorithm can be regarded as only adding texture features of high-resolution data into low-resolution images, so it can keep the spectral characteristics of the original low-resolution images.

\subsection{HPF Transform}

HPF is a high-pass filter fusion algorithm proposed by Schowengerdt in 1980, applying for Landsat MSS images. Chavez et al. (1991) used this algorithm to merge TM,SPOT and aerial images, the formula is as follows:

$B_{H P F_{i}}=\sum_{j} \sum_{k} \frac{B_{\text {low }_{i j k}}+F B_{\text {high }_{j k}}}{2} \quad, i=1, \cdots, 6$

In the formula, $B_{H P F}$ is fusion image transformed by HPF algorithm, and $F B_{\text {high }}$ is high resolution image filtered by high-pass filter, convolution domain select $3 \times 3$ window. The result divided by 2 to avoid excessive expansion of brightness values. This algorithm suppressed low-frequency spectral information and enhanced 
high-frequency spatial information through high-pass filtering, and then High resolution image after the above procedures and low resolution image integrated to increase the spatial resolution.

\subsection{Brovey Transform}

Brovey transform is a ratio fusion algorithm commonly used in multi-spectral image enhancement. It broken down image into two parts, color and brightness, and normalize data by calculate the ratio of each band in RGB composition divided by sum of the three bands, so that maintain the spectral characteristics of low-resolution images, then multiplied ratio result by the high-resolution bands for obtaining high-frequency spatial information. The expression of this fusion method is:

$$
B_{\text {Brovey }_{i}}=\frac{B_{\text {low }_{i}} \cdot B_{\text {high }}}{\sum_{i=1^{\vee}}^{n} B_{\text {low }_{i}}} \quad i=1, \cdots, 6
$$

\subsection{MLT Transform}

MLT is a simple multiplication fusion operation, the integration reflects the mixed messages of low-resolution images and high resolution images, and the formula is expressed as

$$
B_{M L T_{i}}=\sqrt{\sum_{j} \sum_{k} a \cdot B_{l o w_{i j k}} \times b \cdot B_{h i g h} h_{j k}} \quad i=1, \cdots, 6
$$

$B_{M L T}$ is fusion image, As the maximum brightness value achieved by multiplying two band is 65025 in theory, so the square root of product of high and low-resolution images can avoid occur overlarge brightness value. Practical application may need weight different bands for normalization, so $a$ or $b$ has different value.

\section{3. evaluation indicator of fusion result}

Fusion evaluation is an important part in the fusion images process. Currently, there is no uniform methods and standards in multi-source remote sensing image fusion evaluation, Objective and quantitative evaluation is a common method mainly conducted through a variety of statistical methods, and common evaluation indicators include the mean, deviation, information entropy, average gradient and correlation coefficient and so on. Assuming image size is $\mathrm{m} \times \mathrm{n}$, and image gray scale range is $0-255, \mathrm{M}(\mathrm{x}, \mathrm{y})$ and $\mathrm{F}(\mathrm{x}, \mathrm{y})$ represent the low-resolution multi-spectral image and fusion image respectively.

\subsection{Mean}

Mean is the average of pixel gray values, reflecting the average brightness of the image. If the average value is medium, the fusion images have a good visual effect. It is calculated using the following equation:

$$
\bar{M}=\frac{1}{m \times n} \sum_{x=1}^{m} \sum_{y=1}^{n} M(x, y)
$$

\subsection{Deviation}

Deviation refers to the difference between average gray values of original image and average gray values of fused image. Deviation can be expressed as:

$D=\bar{M}(x, y)-\bar{F}(x, y)=\frac{1}{m \times n} \sum_{x=1}^{m} \sum_{y=1}^{n}(M(x, y)-F(x, y))$

Deviation reflects the average change level of spectral characteristics between fusion image and original multi-spectral image. The greater the difference value, the larger the spectrum distortion. 


\subsection{Entropy}

Entropy of a image is an important indicator of measuring abundance of the imagery. The value of image entropy indicates average information content. In general, the greater the entropy, the more abundant information contained in this image. According to principles in Shannon information theory, information entropy of a image $\mathrm{X}$ with 8 bit can be expressed as:

$$
H(x)=-\sum_{i=0}^{255} P_{i} \log _{2} P_{i}
$$

Where $P_{i}$ is the probability of image pixels which gray value is $i$. If the information entropy of fused image is great, representing information content increase than original image, the more abundance information in images, the higher quality of fusion image. So entropy can objectively evaluate changes of information contents.

3.4 Average gradient

The average gradient can be sensitive to reflect contrast of small details in image. It can be used to evaluate the clarity of the image.

$g=\frac{1}{(m-1)(n-1)} \sum_{x=1}^{m-1} \sum_{y=1}^{n-1} \sqrt{\left(\left(\frac{\Delta F_{x}(x, y)}{\Delta x}\right)^{2}+\left(\frac{\Delta F_{y}(x, y)}{\Delta y}\right)^{2} / 2\right.}$

In general, the larger average gradient and the more levels image would result in the more clear fusion image.

\subsection{Correlaion}

Correlation reflects similarity of spectral characteristics between original multi-spectral image and fusion image, this indicator can be expressed as:

$$
\begin{aligned}
& \rho=\sum_{x=1}^{m} \sum_{y=1}^{n}(M(x, y)-\bar{M}(x, y))(F(x, y)-\bar{F}(x, y)) / \\
& \left(\sqrt{\sum_{x=1}^{m} \sum_{y=1}^{n}(M(x, y)-\bar{M}(x, y))^{2}} \sqrt{\sum_{x=1}^{m} \sum_{y=1}^{n}(F(x, y)-\bar{F}(x, y))^{2}}\right.
\end{aligned}
$$

The larger the correlation coefficient, the more the original spectral characteristics of multi-spectral images contained.

\section{4. fusion result and analysis}

Taking southeastern part of Beijing City as the study area which located in the transition zone between urban and rural, having complex land use types such as cropland, grassland, water and buildings, and adopting ETM+ PAN and multispectral images obtained on September 6, 2006 as data, fusion bands of the multispectral images select b3,b4 and b5. Applying the four fusion algorithms mentioned above to carry out fusion experiment, and fusion results are shown in Fig.1. 


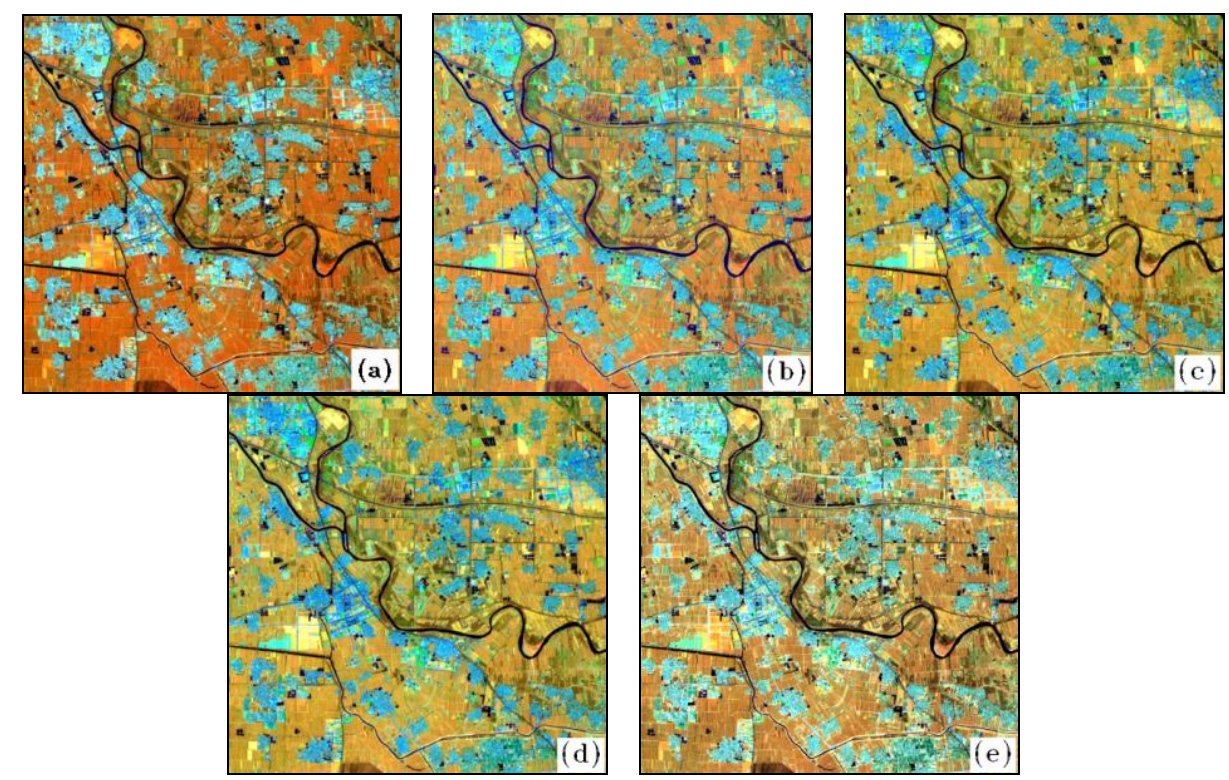

(a)original image; (b)SFIM-fused image; (c)HPF-fused image; (d)Brovey-fused image; (e)MLT-fused image

Fig.1. Four fused images and original ETM+ image (RGB:453)

The mean, deviation, entropy, average gradient, correlation in each band were calculated from the original ETM+ image and the four fused images for objective and quantitative evaluation. The details of calculation results are given in Table 1.

Table 1. Statistical parameters of the four fused images and the original ETM+ multispectral image

\begin{tabular}{|l|l|l|l|l|c|c|}
\hline Image & Band & Mean & Deviation & Entropy & Average gradient & Correlation \\
\hline Original & R & 182.272 & & 4.065 & 8.237 & \\
& G & 171.156 & & 5.685 & 14.645 & \\
& B & 154.113 & & 5.190 & 14.192 & \\
\hline SFIM & R & 182.341 & -0.062 & 4.067 & 5.789 & 0.989 \\
& G & 171.278 & -0.122 & 5.746 & 23.691 & 0.949 \\
& B & 156.625 & -2.547 & 5.179 & 22.226 & 0.918 \\
\hline HPF & R & 169.186 & 13.068 & 5.472 & 26.023 & 0.356 \\
& G & 144.956 & 26.2 & 7.345 & 21.014 & 0.942 \\
& B & 141.035 & 13.078 & 6.953 & 20.568 & 0.917 \\
\hline Brovey & R & 180.117 & 2.155 & 3.945 & 32.504 & 0.351 \\
& G & 168.578 & 2.578 & 4.561 & 16.978 & 0.959 \\
& B & 164.967 & -10.854 & 3.977 & 19.127 & 0.927 \\
\hline MLT & R & 178.278 & 3.944 & 3.987 & 8.608 & 0.977 \\
& G & 167.453 & 3.703 & 5.824 & 23.244 & 0.913 \\
& B & 154.167 & -0.05 & 5.237 & 21.832 & 0.874 \\
\hline
\end{tabular}


According to the statistical parameters in the Table 1, It is found that HPF transform makes the mean of each band significantly reduced, so fused image becomes dark; and deviation in HPF transform is also biggest, second is Brovey transform, SFIM transform is least. Therefore, spectral distortion of HPF is most serious, second is Brovey, MLT is third, and SFIM possess the best spectral fidelidy. The situation of correlation opposites to the deviation, entropy represent information content and quality of the fused image. Exclude Brovey transform, each band of images generated through other algorithms has increased. The increment of entropy in HPF transform is significant; Form the result of average gradient, only red band of Brovey decrease, the rest increase significantly. Average gradient indicate involvement of high-frequency spatial information to some extent, SFIM has highest average gradient, followed HPF and MLT, Brovey is the lowest.

\section{Conclusion and recommendation}

From the visual interpretation, spatial resolution and clarity of four images derived from four fusion algorithms have greatly improved comparing with original ETM+ images. The results of this study show that different resolution images from the same sensor system can avoid errors due to free of the seasonal difference, various solar illumination and other environmental condition differences, and co-registration errors, which are common in heterogeneous sensor. The quality of fusion image is affected by spectral fidelity and involvement of high-frequency spatial information. Through comparison of the above methods, it is obvious that SFIM algorithm is the best method in retaining spectral information of original image, which does not cause spectral distortion, and has highest spatial frequency information gain for the ETM+ images in the study area. If apply fusion images created by this algorithm to research classification, the accuracy of automatic classification may be improved.

\section{References}

[1] Liu J G. Evaluation of Landsat7 ETM+ Panchromaic Band for Image fusion with Multispectral Bands[J]. Natural Resources Research, 2000，9(4): 269-276.

[2] Chavez P S Jr, Sliders S C, Anderson J A.Comparion of Three Different Methods to Merge Multiresolution Data: Landsat TM and SPOT Panchromatic[J]. Photogrammetric Engineering and Remote Sensing, 1991, 57(3): 295-303

[3] Pohl C, Genderen J L van. Multisensor Image Fusion in Remote Sensing: Concepts, Methods and Applications[J]. International Journal of Remote Sensing, 1998， 19(5): 823-854

[4] Zhao Y S, Li J W. A Study on Land Use Classification Using ETM+[J]. Remote Sensing Information (in Chinese), 2001, (2): 39-42.

[5] Chen D C, Zhou H B, Chen Z Y et al. Study on Fusion Algorithms of TM and SPOT images[J]. Remote Sensing Technology and Application (in Chinese), 2001, 16(2): 110-115.

[6] Liu J G. Smoothing Filter-based Intensity Modulation: a Spectral Preserve Image Fusion Technique for Improving Spatial Details[J]. International Journal of Remote Sensing, 2000， 21(18): 3461-3472

[7] Li Junjie, He Longhua, Dai Jinfang. Analysis of Pixel-level Remote Sensing Image Fusion Methods[J].Geo-information Science (in Chinese), 2008, 10(1): 128-134.

[8] Xu Han-qiu. Study on Data Fusion and Classification of Landsat 7 ETM+ Imagery[J]. Journal of Remote Sensing(in Chinese), 2005, 9(2): 186-194. 